\title{
Spots on prevention of emergence delirium and agitation in children using dexmedetomidine
}

Volume 8 Issue 3 - 2017

\section{Introduction}

Eckenhoff et al. (1960), who first described emergence delirium (ED) as a post-surgical phenomenon. They studied over 14,000 patients who experienced unusual post-operative behavioral disturbances. ${ }^{1}$ Some authors defined ED as a dissociated state of consciousness in which the child is irritable, uncooperative, incoherent, and inconsolably crying, moaning, kicking, or thrashing. These children do not recognize or identify familiar objects or people. Combative behavior has been more often described than simple restlessness and incoherence. The general incidence of ED ranges from $10 \%$ to $50 \%$, However it can be as high as $80 \% .^{2}$ It's not a simple phenomenon as it can result in injury to the children themselves or damage to the surgical site, leading to dissatisfaction and anxiety for the parents and requiring extra nursing care, which further increases associated healthcare costs. ${ }^{3}$

There is no definitive explanation for emergence agitation. Many different causes have been suggested, such as rapid return of consciousness in an unfamiliar environment, the presence of pain (wounds, sore throat, bladder distension, etc.), stressful induction, airway obstructions, a noisy environment, the duration of anesthesia, the child's personality, anesthetic premedication and the anesthetic technique used. The most important risk factors are the use of the relatively insoluble halogenated inhaled anesthetics, such as sevoflurane or desflurane. These agents have been postulated to alter brain activity by interfering with the balance between neuronal synaptic inhibition and excitation in the central nervous system. The difficulty with establishing etiology explains the major variation in the prevalence rates reported in the literature. ${ }^{4-6}$

A range of factors may have an impact on the postoperative behavior of patients, as will be described below.

a. Age ( 2 to 4 years of age)

b. Psychological, social and environmental factors (impulsive, emotional, less sociable children and anxious parents)

c. Postoperative pain

d. Type of surgery (Oto-rhino-laryngological surgical procedures, and ophthalmological operations)

e. Inhaled anesthetics

f. Adjuvant drugs (midazolam, ketamine, nalbuphine, clonidine, fentanyl, propofol, and dexmedetomidine) ${ }^{4}$

There are no widely accepted assessment scales for the evaluation of EA in children, which led to the use of numerous assessment methods and agitation rating systems. In most trials, the Pediatric Anesthesia Emergence Delirium scale (PAED) was used for assessing the incidence of ED. It consists of five points scale; $1=$ the child makes eye contact with the caregiver, $2=$ the child's actions are purposeful, $3=$ the child is aware of his/her surroundings, $4=$ the

\author{
Michael R EIMansoury \\ Fujairah Hospital, UAE
}

Correspondence: Michael R ElMansoury, Fujairah Hospital, UAE, Email michaelelmansoury@gmail.com

Received: July 0I, 2017 | Published: July 10, 2017 child is restless, and $5=$ the child is inconsolable. ${ }^{7}$ Aono et al. (1997), developed four points scale; $1=$ calm; $2=$ not calm but could be easily consoled; $3=$ moderately agitated or restless and not easily calmed; $4=$ combative, excited, or disoriented, thrashing around. Scores of one and two were considered as absence of emergence delirium, and scores of three and four were analyzed as presence of EA. ${ }^{8}$ Cole et al. (2002), developed 5 points agitation scale; $1=$ Sleeping, $2=$ Awake, calm, $3=$ Irritable, crying, 4= Inconsolable crying, and 5= Severe restlessness, disorientation. ${ }^{9}$ There are certain limitations regarding ED scales have been identified. The principal one is the difficulty in differentiating post-anesthetic agitation from the symptoms of postoperative pain. It's of great benefit if the postoperative pain has been ruled out as the cause of postoperative agitation. It is possible that the adoption of an age-based pain scale in combination with the PAED scale could be useful for defining diagnosis and assessment of ED. ${ }^{4}$

Dexmedetomidine is an $\alpha 2$-adrenoceptor agonist with approval from the Food and Drug Administration (FDA) for sedation in the ICU, and in patients before and during surgical and other procedures. ${ }^{10,11}$ Dexmedetomidine is one of the imidazole $\alpha 2$ adrenergic agonists subclass which exhibits a high ratio of specificity for the $\alpha 2$ versus the $\alpha 1$ receptor. Dexmedetomidine exhibits an $\alpha 2: \alpha 1$ specificity ratio of 1600:1 thereby making it a complete agonist at the $\alpha 2$-adrenergic receptor. Dexmedetomidine has a short half-life (2-3 hours) and is commercially available for intravenous administration. ${ }^{12}$ Dexmedetomidine's pharmacokinetic profile includes a rapid distribution phase (distribution half-life of 6 minutes), and elimination half-life of 2 hours. Dexmedetomidine is $94 \%$ protein bound to serum albumin and $\alpha 1$-glycoprotein. It undergoes hepatic metabolism with limited unchanged drug excreted in the urine or stool. ${ }^{12}$

Dexmedetomidine physiologic effects are mediated via postsynaptic $\alpha 2$-adrenergic receptors. Centrally acting $\alpha 2$-adrenergic agonists also activate receptors in the medullary vasomotor center reducing norepinephrine with a resultant central sympatholytic effect leading to decreased heart rate (HR) and blood pressure (BP). 
Central CNS stimulation of parasympathetic outflow and inhibition of sympathetic outflow from the locus cereleus in the brainstem play a prominent role in the sedation and anxiolysis produced by these agents. Decreased noradrenergic output from the locus cereleus allows for increased firing of inhibitory neurons including gamma amino butyric acid (GABA). Primary analgesic effects and potentiation of opioidinduced analgesia result from the activation of $\alpha 2$-adrenergic receptors in the dorsal horn of the spinal cord and the inhibition of substance $\mathrm{P}$ release. ${ }^{12}$ Dexmedetomidine have a unique pharmacologic profile as it can produce stage II non-rapid eye movement sleep through activation of the endogenous sleep promoting pathway, which induce conscious sedation. ${ }^{3}$

Zhu M et al. (2015), did a meta-analysis which included 20 randomized controlled trials published before October 2014. They studied intervention, premedication, surgery procedures, anesthesia induction and maintenance of anesthesia related to emergence delirium. They found that there was no significant difference in the incidence of ED between dexmedetomidine and fentanyl. They reported that dexmedetomidine had no significant impact on the incidence of emergence delirium in comparison to midazolam. However, they advised to interpret these findings cautiously due to small number of studies. They concluded that dexmedetomidine delay emergence time, time to extubation, and time to discharge from post anesthesia care unit (PACU). On the other side, they demonstrated that dexmedetomidine has a beneficial effect in decreasing post-operative nausea and vomiting (PONV), and number of patients requiring analgesic. More importantly, they showed that dexmedetomidine had identical analgesic effects compared with fentanyl. They used a variety of doses as bolus ranged from $0.15-1 \mathrm{mcg} / \mathrm{kg} \mathrm{IV}, 2.5-4 \mathrm{mcg} / \mathrm{kg}$ orally, and $1,2 \mathrm{mcg} / \mathrm{kg}$ in caudal, and fascia iliaca blocks respectively. ${ }^{3}$

Equivalent results were seen in a recent meta-analysis by Viswanath $\mathrm{O}$ et al. (2015). They evaluated 45 articles published from 1999 to 2015. They found that dexmedetomidine one of the drugs that significantly reduced the risk of ED was associated with a decrease in the risk of postoperative nausea and vomiting. Dexmedetomidine exhibited significantly longer emergency response time compared to placebo. ${ }^{1}$ Both meta-analysis didn't mention about possible adverse effects regarding hemodynamic consequences of intravenous dexmedetomidine. However Mahmoud $\mathrm{M}$ et al., mentioned that heart rate responses are rarely of clinical significance, and they do not usually warrant treatment. Extreme bradycardia can occur if dexmedetomidine is administered to a patient receiving digoxin, $\beta$-adrenergic blockers, calcium channel blockers, and others. Although decreases in HR and a biphasic effect on BP are observed with increasing doses of dexmedetomidine. ${ }^{13}$

\section{Conclusion}

In conclusion, ED is common terrifying complication in anesthetized preschool children, especially when using sevoflurane. According to the currently available literature, intra-operative administration of dexmedetomidine either at the end of surgery or continuously as an anesthetic agent has been found superior to other agents in decreasing the incidence of ED. Dexmedetomidine has a unique pharmacologically characteristic to have safer sedative and analgesic effects without risk of respiratory depression. In addition to, it decreases incidence of PONV. However, there was a significant increase in emergence time, time to extubation and discharge from PACU. Further clinical trials need to be conducted to validate the proper dose and their relationship with delayed emergence time and discharge from PACU. Moreover, further research is needed to assure timing and methods of administration of dexmedetomidine as an effective drug to prevent emergence delirium in pediatrics anesthesia. In addition to a widely accepted model for emergence delirium scale need to be employed.

\section{Acknowledgments}

None.

\section{Conflicts of interest}

Author declares that there is no conflict of interest.

\section{References}

1. Viswanath O, Kerner B, Jean Y, et al. Emergence delirium: A narrative review. J Anesth Clin Sci. 2015;4(2):2049-9752.

2. Vlajkovic GP, Sindjelic RP. Emergence delirium in children: Many questions, few answers. Anesth Analg. 2007;104(1):84-91.

3. Zhu M, Wang $\mathrm{H}$, Zhu A, et al. Meta-analysis of dexmedetomidine on emergence agitation and recovery profiles in children after sevoflurane anesthesia: different administration and different dosage. PLoS One. 2015;10(4)

4. Silva LM, Braz LG, Módolo NS. Emergence agitation in pediatric anesthesia: current features. J Pediatr (Rio J). 2008;84(2): 107-113.

5. Yli-Hankala A, Vakkuri A, Särkelä $M$, et al. Epileptiform electroencephalogram during mask induction of anesthesia with sevoflurane. Anesthesiology. 1999;91(6):1596-603.

6. Kain ZN, Caldwell-Andrews AA, Maranets I, et al. Preoperative anxiety and emergence delirium and postoperative maladaptive behaviors. Anesth Analg. 2004;99(6):1648-1654.

7. Sikich N, Lerman J. Development and psychometric evaluation of the pediatric anesthesia emergence delirium scale. Anesthesiology. 2004;100(5):1138-1145.

8. Aono J, Ueda W, Mamiya K, et al. Greater incidence of delirium during recovery from sevoflurane anesthesia in preschool boys. Anesthesiology. 1997;87(6):1298-1300.

9. Cole JW, Murray DJ, McAllister JD, et al. Emergence behaviour in children: defining the incidence of excitement and agitation following anaesthesia. Paediatr Anaesth. 2002;12(5):442-447.

10. Marhofer P, Brummett CM. Safety and efficiency of dexmedetomidine as adjuvant to local anesthetics. Curr Opin Anaesthesiol. 2016;29(5):632-637.

11. Wang W, Huang P, Gao W, et al. Efficacy and Acceptability of Different Auxiliary Drugs in Pediatric Sevoflurane Anesthesia: A Network Metaanalysis of Mixed Treatment Comparisons. Sci Rep. 2016;6:36553.

12. Tobias JD. Dexmedetomidine: Applications in pediatric critical care and pediatric anesthesiology. Pediatr Crit Care Med. 2007;8(2):115-131.

13. Mahmoud M, Mason KP. Dexmedetomidine: review, update, and future considerations of paediatric perioperative and periprocedural applications and limitations. Br J Anaesth. 2015;115(2):171-182. 